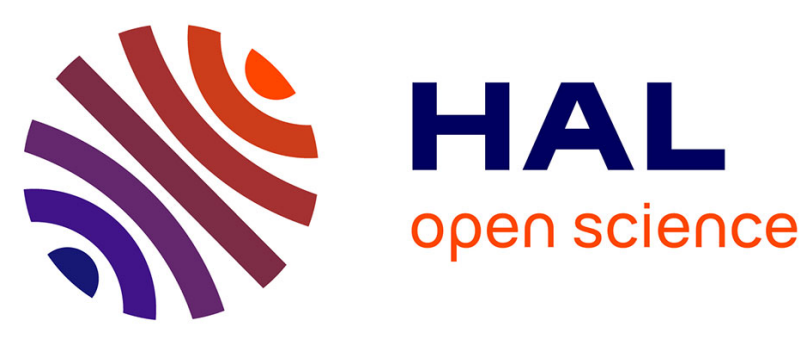

\title{
L'optique dans l'ordinateur
}

Pierre Chavel, S. Laval, Jean Taboury

\section{To cite this version:}

Pierre Chavel, S. Laval, Jean Taboury. L'optique dans l'ordinateur. Revue de Physique Appliquée, 1987, 22 (10), pp.1215-1219. 10.1051/rphysap:0198700220100121500 . jpa-00245671

\section{HAL Id: jpa-00245671 https://hal.science/jpa-00245671}

Submitted on 1 Jan 1987

HAL is a multi-disciplinary open access archive for the deposit and dissemination of scientific research documents, whether they are published or not. The documents may come from teaching and research institutions in France or abroad, or from public or private research centers.
L'archive ouverte pluridisciplinaire HAL, est destinée au dépôt et à la diffusion de documents scientifiques de niveau recherche, publiés ou non, émanant des établissements d'enseignement et de recherche français ou étrangers, des laboratoires publics ou privés. 


\title{
L'optique dans l'ordinateur
}

\author{
P. Chavel $\left({ }^{*}\right)$, S. Laval $\left({ }^{* *}\right)$ et J. Taboury $(*)$ \\ (*) Institut d'Optique, Laboratoire Associé au C.N.R.S., B.P. 43, 91406 Orsay Cedex, France \\ (**) Institut d'Electronique Fondamentale, Laboratoire Associé au C.N.R.S., Université de Paris-Sud, \\ bâtiment 220, 91405 Orsay Cedex, France
}

(Reçu le 10 mars 1987, accepté le 22 juin 1987)

\begin{abstract}
Résumé. - Il existe aujourd'hui de sérieux espoirs d'augmenter la capacité de calcul des ordinateurs et des processeurs parallèles à l'aide de faisceaux lumineux chargés non seulement du transport, mais aussi du traitement de l'information à l'intérieur même des processeurs grâce à des interactions non linéaires appropriées avec la matière. Ce numéro spécial de la Revue de Physique Appliquée est en grande partie constitué à partir de communications présentées lors de la « seconde journée d'étude sur les fonctions optiques dans l'ordinateur », organisée à Orsay par la Société Française d'Optique le 17 septembre 1986. Ce bref article d'introduction présente l'ensemble d'un domaine nouveau auquel la coopération de trois thèmes scientifiques est en train de donner naissance : la physique des matériaux optiques non linéaires, le traitement optique de l'information et l'architecture des ordinateurs doivent en effet réunir leurs efforts pour faire de « l'optique dans l'ordinateur » une réalité.
\end{abstract}

\begin{abstract}
Light beams capable not only of carrying information from one place to another, but also of processing it by suitable nonlinear interaction with matter inside digital processors are likely to foster an increase in power of computers. This special issue of Revue de Physique Appliquée is to a large extent based on communications presented at the "seconde journée d'étude sur les fonctions optiques dans l'ordinateur », organized in Orsay, France, by the French Optical Society, September 17, 1986. The present short introductory article surveys the new domain resulting of the interaction between three scientific areas : nonlinear optical materials physics, optical information processing and computer architecture have to cooperate for « optical computing » to become a reality.
\end{abstract}

Avec les imprimantes à laser, les nouvelles familles de photorécepteurs, les communications par fibres optiques entre ordinateurs et les mémoires de masse à disques optiques numériques, l'optique, par l'optoélectronique, a déjà pénétré la technologie de l'informatique et s'est ainsi rapproché des ordinateurs. Cependant, il semble raisonnable de s'attendre à un pas de plus: l'optique pourrait entrer à l'intérieur même des processeurs, soit pour se charger d'une partie des connexions, soit pour effectuer des calculs. Deux apports fondamentaux peuvent justifier une telle évolution : d'une part, l'interaction non linéaire entre lumière et matière fait intervenir des temps caractéristiques parfois inférieurs à la picoseconde, si bien que l'optique pourrait apporter une plus grande rapidité ; d'autre part, en l'absence de matière, les faisceaux lumineux se croisent sans interagir, si bien que l'optique, grâce à un niveau de parallélisme jusqu'à présent inaccessible, pourrait apporter une plus grande densité d'interconnexions et de ce fait rendre possibles de nouvelles architectures parallèles.

Le présent numéro spécial est en grande partie constitué par la remise en forme de communications présentées à Orsay le 17 septembre 1986 au cours de la Deuxième Journée d'Etudes sur l'Optique dans l'Ordinateur organisée par la Société Française d'Optique (S.F.O.) avec les copatronages de la Société Française de Physique et de la Société des Electriciens et Electroniciens et avec l'aide de l'Institut d'Electronique Fondamentale et de l'Institut d'Optique. Le programme de cette journée mettait l'accent sur les matériaux présentant de fortes nonlinéarités et leurs applications ; esquissons dans cette introduction un tableau de l'ensemble du domaine de «l'optique dans l'ordinateur», en commençant par un aperçu historique. 
1. Bistabilité optique et traitement optique de l'information.

C'est à Gibbs et à son équipe [1] qu'est due l'observation, en 1976, de la bistabilité dans une cavité optique résonnante contenant un matériau non linéaire, en l'occurrence de la vapeur de sodium. On sait qu'on entend par bistabilité l'existence de deux états stables pour un système pour certaines valeurs données aux paramètres : dans le cas optique, c'est le plus souvent de deux états différant par leur coefficient de transmission ou de réflexion qu'il s'agit. La bistabilité résulte de la conjonction entre non-linéarité et rétroaction. La rétroaction peut être effectuée par voie externe à l'aide d'un signal électrique intermédiaire, par les réflexions multiples dans une cavité résonnante, ou parfois directement par une combinaison d'effets non linéaires du matériau, comme dans le composant SEED [2]. Venant à la suite de plusieurs travaux précurseurs, les travaux de l'équipe de Gibbs à Tucson, ceux du laboratoire dirigé par S. D. Smith à Edimbourg, et les succès obtenus aux laboratoires Bell ont largement contribué au développement des études sur la bistabilité optique, qui connaissent depuis lors un essor continu soutenu par deux motivations : d'une part, le désir de voir apparaître des composants optiques bistables utilisables pour le traitement binaire de l'information, d'autre part le souhait de parvenir à une meilleure compréhension fondamentale des processus dynamiques non linéaires. Nous reviendrons sur le premier de ces points. La référence [3], constituée par les actes d'un colloque récent, donne une idée du champ couvert par le sujet et de sa vitalité.

C'est dans une communauté scientifique toute différente qu'est apparue l'expression « traitement optique de l'information ». Sous sa forme initiale, il s'agit en effet de l'association du concept de filtrage des fréquences spatiales et du laser. Duffieux [4] avait montré qu'avec une bonne approximation, tout système d'imagerie peut être décrit dans le formalisme du filtrage linéaire homogène, c'est-à-dire qu'il effectue une convolution sur les données bidimensionnelles que constitue son plan objet, Maréchal et Croce [5] avaient suggéré d'utiliser cette propriété pour le traitement des images. Le laser, associé à l'holographie dont il rend la pratique aisée, a permis dès les années 1960 de réaliser une grande variété de convolutions optiques. A une époque où les ordinateurs n'avaient pas la capacité de traiter les images, le traitement optique analogique semblait une voie pleine d'avenir. Son succès le plus remarquable est le développement du corrélateur pour radar à vision latérale [6], capable de transformer les données brutes inintelligibles du radar à synthèse d'ouverture en images d'excellente qualité. Ce calculateur spécialisé, utilisé sous sa forme originale de 1963 à 1982 environ, traite en parallèle plus de $10^{6}$ données. Il souffre cependant des défauts typiques de sa génération : le traitement y est analogique; les opérations aisément réalisables se limitent à l'addition, à la multiplication, à la convolution et à la transformation de Fourier, ce qui n'est acceptable que pour un processeur spécialisé ; enfin et surtout, le défaut de composant capable d'enregistrer en temps réel l'image à traiter imposait le recours à un intermédiaire photographique. Il existe aujourd'hui de bons espoirs de surmonter ces obstacles.

Si l'optique non linéaire et le filtrage des fréquences spatiales n'ont guère de points communs au départ, ils se rencontrent aujourd'hui dans leur volonté de faire entrer l'optique dans l'ordinateur. Ce domaine d'application commun peut devenir fédérateur. Citons les progrès qu'il a suscités ces dernières années tant du côté des matériaux et composants que de celui des «architectures de systèmes optiques ».

\section{Matériaux et composants non linéaires.}

L'identification des classes de matériaux présentant de fortes non-linéarités optiques est une première voie de recherche, qui ne saurait être séparée de l'étude des mécanismes mis en jeu; à cela doit s'ajouter le souci de quelques contraintes pratiques : les propriétés physico-chimiques des composés utilisés doivent être compatibles avec des conditions d'utilisation raisonnables et doivent potentiellement se prêter à la mise au point de technologies de production en grande quantité. Les principales approches actuelles concernent les semi-conducteurs : soit ceux de la famille III-V, massifs $[7,8]$ ou en puits quantiques multiples [9], soit ceux de la famille II-VI [10, 11], ou encore le silicium [12] ; mais il convient de citer également les matériaux photoréfractifs, qui, outre certains semi-conducteurs déjà cités, comprennent en particulier les cristaux de niobate de lithium et les oxydes de bismuth-silicium et de bismuth-germanium ; de plus, certains espoirs sont mis dans les matériaux organiques à électrons $\pi$ délocalisés.

Les quatre premiers articles de ce numéro spécial concernent de telles études de matériaux : J. Messier, P. A. Chollet, F. Kajzar, J.-M. Nunzi et D. Grec passent en revue les effets de non-linéarités cubiques dans les milieux organiques, alors que $\mathrm{J}$. Badan, D. Josse, I. Ledoux, P. Vidakovic et J. Zyss s'attachent à identifier les matériaux organiques présentant de fortes non-linéarités quadratiques. J. P. Pocholle, M. Razeghi, J. Raffy, M. Papuchon, C. Weisbuch, C. Puech, L. Heinrich et J. E. Vimont présentent des résultats expérimentaux sur les nonlinéarités excitoniques dans des puits quantiques InGaAs/InP. Enfin, G. Roosen, A. Brun, G. Le Saux et G. Pauliat présentent une synthèse sur les phénomènes physiques mis en jeu dans les 
matériaux photoréfractifs et évoquent leurs utilisations possibles.

Précisément, l'application des non-linéarités au calcul par voie optique est une voie de recherche active : à côté des bistables optiques au sens propre, plusieurs catégories de dispositifs non linéaires méritent attention. C'est le cas des composants à caractéristique non bistable mais fortement non linéaire, parfois appelés «transphaseurs », par analogie avec les transistors, s'ils présentent une région à fort gain ; une caractéristique adaptée à l'opération logique booléenne NOR, par exemple, paraît fort intéressante. Citons aussi la conjugaison de phase. Pour se révéler utiles en pratique, ces dispositifs doivent réunir potentiellement au moins certains des avantages suivants : se prêter à une intégration assez élevée, répondre en des temps très brefs, si possible de l'ordre de la picoseconde, à une excitation aussi bien qu'à l'arrêt de cette excitation, différencier le faisceau de lecture et le faisceau d'écriture, dissiper une quantité d'énergie compatible avec la nécessité de l'évacuation thermique aux taux de répétition élevés.

C'est dans cette lignée que se situent cinq des articles des pages qui suivent : $\mathrm{H}$. Chelli, A. Koster, N. Paraire, F. Pardo, H. Sauer, M. Carton et S. Laval utilisent les non-linéarités ultra-rapides du silicium en film mince pour commuter un faisceau lumineux ; D. Hulin, A. Antonetti, M. Joffre, A. Migus, A. Mysyrowicz, N. Peyghambarian et H. M. Gibbs présentent l'obtention d'excitation et de désexcitation subpicoseconde par effet Stark dans des puits quantiques multiples; J. Collet et $T$. Amant proposent une version nouvelle de porte NOR dans un semi-conducteur II-VI ; J. Kuszelewicz et J. L. Oudar soulignent l'intérêt des bistables optiques actifs obtenus par cavités laser à semiconducteur couplées : B. Thédrez et R. Frey proposent l'utilisation du mélange à quatre ondes pour réaliser des opérations booléennes.

A côté des études de base qui viennent d'être évoquées existent d'ores et déjà un certain nombre de développements technologiques pris en charge par les laboratoires de recherche et de développement dans l'industrie : on voit apparaître, sous les noms (peu satisfaisants) de modulateurs spatiaux de lumière ou de valves optiques toute une série de composants compatibles avec les technologies actuelles et capables de moduler la transmission ou la réflexion d'une image en réponse à une commande optique ou électrique : le successeur de la plaque photographique pour le traitement du signal existe donc aussi à ce stade, et il est même dans quelques cas disponible commercialement. Si les performances actuelles sont loin des espoirs suscités par les recherches citées plus haut, l'existence même de tels outils est un atout précieux pour la recherche sur les « architectures » de systèmes optiques.
L'article de D. Comte, P. Siron, X. Thibault, P. Churoux, M. Fracès et M. Laug décrit une utilisation expérimentale d'une valve optique commerciale pour la connexion optique dynamique en «crossbar » entre les éléments d'un réseau de processeurs parallèles. La synthèse présentée par G. Lebreton montre l'ensemble des tendances d'évolution des valves optiques.

\section{Architectures de systèmes optiques.}

Bistables et commutateurs optiques, éléments pour mixage d'ondes et génération d'harmoniques, oscillateurs paramétriques, valves optiques, hologrammes réinscriptibles ou fixes, fibres et guides passifs ou non linéaires, lentilles, prismes, réseaux, miroirs, déflecteurs et modulateurs électro et acousto-optiques, ... : tous ces composants, les uns en cours d'études et les autres classiques et bien connus, sont les ingrédients dont dispose ou disposera bientôt l'opticien dans sa tâche nouvelle d'architecte de processeurs optiques. Pragmatique, il peut chercher à établir des communications optiques à l'intérieur des processeurs; attaché aux valeurs éprouvées, il peut recourir à la transformation de Fourier optique sur des signaux analogiques. Mais s'il se montre plus audacieux et plus novateur, il peut explorer de nombreuses voies nouvelles. Le numéro spécial de juillet 1984 de I.E.E.E. Proceedings [13] reste une référence de base pour ces dernières, mais les progrès ont été suffisamment rapides depuis lors pour qu'il soit nécessaire de citer aussi plusieurs autres publications plus récentes : les références [1419] fournissent un échantillonnage représentatif du domaine. Contentons-nous ici d'en évoquer les lignes directrices.

Un des cas le plus spectaculaire d'application de la transformée de Fourier optique est constitué par les analyseurs de spectres unidimensionnels obtenus par diffraction de la lumière par une onde acoustique modulée par le signal analysé et traversant un cristal ou une cuve acousto-optique. Ces composants, disponibles commercialement, sont actuellement les seuls à opérer une transformation de Fourier ou une convolution sur environ 1000 points avec des cadences d'entrée des données de l'ordre de $1 \mathrm{GHz}$ et illustrent bien le parallélisme du traitement optique.

Tant qu'il est linéaire, un système optique peut automatiquement être interprété comme un multiplicateur de matrices. Il est performant dès lors que l'on sait imposer la réponse au signal d'entrée en chaque point. Le système est alors caractérisé par une matrice. Au cas habituel des systèmes invariants par translation, décrits formellement par des matrices de Toeplitz, sont venus s'ajouter depuis les travaux de Goodman [20] de nombreux schémas de multiplicateurs de matrices optiques. Plusieurs d'entre eux [13], utilisant des déflecteurs acoustooptiques, peuvent être interprétés comme des pro- 
cesseurs optiques «systoliques », au sens où ce mot est utilisé dans le domaine du calcul parallèle [21]. Comme d'ailleurs tout schéma d'interconnexion peut être mis sous la forme d'une matrice dont le produit par la matrice colonne des signaux d'entrée donne la matrice colonne des signaux de sortie, chaque connexion étant représentée par un 1 dans la matrice des connexions, les connexions optiques elles-mêmes peuvent être coulées dans ce moule. On y trouve donc les réseaux de connexions « crossbar » tels que celui décrit par $M$. Comte et ses collègues dans ce numéro, mais aussi des schémas plus spécialisés comme le "perfect shuffle », utile pour paralléliser certains algorithmes nécessitant des connexions à distance arbitraire et dont une version optique a été proposée récemment [22].

Si les transformations de Fourier et les produits de matrices restent du domaine de l'analogique, les opticiens se penchent également sur la façon d'introduire le calcul binaire dans leur domaine. Signalons d'abord l'extension du produit de matrices acoustooptiques à la multiplication binaire par convolution [23]. Mais surtout, l'introduction des composants bistables et des opérateurs booléens optiques non linéaires a fait apparaître toute une série d'idées dont l'opération peut être décrite comme un processeur cellulaire optique : comme dans un automate cellulaire, chaque élément du processeur effectue une opération booléenne à plusieurs entrées sur les signaux binaires délivrés par certains de ses voisins et transmet à son tour le résultat à d'autres voisins, en général à travers une boucle de rétroaction qui le renvoie à l'entrée du composant non linéaire. La puissance théorique de tels schémas, illustrée dans les numéros spéciaux déjà cités par des exemples de calcul numérique et de traitement d'image, est considérable [24].

Enfin, les architectures dites « connexionnistes » fournissent une autre illustration prometteuse des possibilités de l'approche optique : dérivées des machines associatives et de certains modèles neuro- naux [25], elles consistent à envoyer à l'entrée de chaque élément de calcul $N$ (appelé, de façon quelque peu abusive, "neurone ») une stimulation provenant d'un nombre élevé de neurones voisins ou non, les uns excitateurs, les autres inhibiteurs. L'état, binaire, du neurone $N$ résulte d'un seuillage effectué sur la stimulation totale. De tels schémas peuvent fonctionner comme machines associatives, ainsi que le montre l'article de A. Maruani et G. Sirat dans ce numéro, mais aussi comme processeurs spécialisés dans des problèmes d'optimisation particulièrement lourds en calcul. Depuis la première version optique [26], de nombreuses variantes ont été proposées et mettent en valeur les propriétés intéressantes de ces architectures particulièrement adaptées aux possibilités actuelles de l'optique en raison de l'importance des connexions et de la coexistence de signaux binaires et de signaux analogiques. On peut dire que la distinction entre connexions et éléments de calcul, si évidente dans les processeurs électroniques d'aujourd'hui, perd de sa clarté avec de telles approches et c'est peut-être au prix de pareilles évolutions conceptuelles que l'optique peut efficacement entrer dans l'ordinateur.

Cette introduction, ainsi que les articles qui suivent, montrent combien les approches de «l'optique dans l'ordinateur » sont diverses et font intervenir des disciplines peu habituées à collaborer : il reste sans doute fort à faire pour que chacun parle le même langage et ait transmis son message efficacement à ses collègues. Tous les pays engagés fortement dans la recherche s'y efforcent. Puisse ce numéro spécial y contribuer pour sa part. Nous sommes reconnaissants à la Revue de Physique Appliquée de lui avoir ouvert ses colonnes, à M. J. Jerphagnon qui nous a incités à élaborer ce numéro spécial, et nous remercions pour leur aide efficace nos collègues qui ont fait partie du comité d'organisation de la Journée d'Etudes S.F.O. sur l'Optique dans l'Ordinateur et du comité de lecture pour les articles que l'on va lire.

\section{Bibliographie}

[1] Gibbs, H. M., McCall, S. L. and Venkatesan, T. N. C., Phys. Rev. Lett. 36 (1976) 113.

[2] Miller, D. A. B., Chemla, D. S., Damen, T. C., Gossard, A. C., Wiegman, W., WoOd, T. H. and Burrus, C. A., Appl. Phys. Let. 45 (1984) 13.

[3] Optical Bistability III, H. M. Gibbs, P. Mandel, N. Peyghambarian and S. D. Smith, editors (Springer, Berlin) 1986.

[4] Duffieux, P. M., L'intégrale de Fourier et son application à l'optique, chez l'auteur, Besançon, $1946 ; 2^{\mathrm{e}}$ édition (Masson, Paris) 1976.

[5] Maréchal, A. et Croce, P., C. R. Hebd. Séan. Acad. Sci. 237 (1953) 607.
[6] Cutrona, L., Leith, E., Porcello, L. J. and Vivian, W. E., Proc. I.E.E.E. 54 (1966) 1026.

[7] Gibbs, H. M., McCall, S. L., Venkatesan, T. N. C., Gossard, A. C., Passner, A., Wiegman, W., Appl. Phys. Let. 35 (1979) 6.

[8] Miller, D. A. B., SMith, S. D. and Johnston, A. M., Appl. Phys. Let. 35 (1979) 658.

[9] Miller, D. A. B., Gossard, A. C. and Wiegman, W., Opt. Let. 9 (1984) 162.

[10] Smith, S. D., Mathew, J. G. H., Taghizadeh, M. R., WAlker, A. C., Wherrett, B. S., Hendry, A., Opt. Commun. 51 (1985) 357.

[11] Bigot, J. Y., FRINDI, M., WEgener, M., Hönerlage, B., Lévy, R. et Grun, J. B., p. 132 de la référence 3. 
[12] Pardo, F., Koster, A., Chelli, H., Paraire, N. et LAVAL, S., p. 83 de la référence 3.

[13] Special Issue on Optical Computing, Proc. I.E.E.E. 72 (1984) 755-978.

[14] Special Issue on Optical Computing and Optical Information Processing Components, Opt. Eng. 24 (1985) 1-149.

[15] Special Issue on Digital Optical Computing, Opt. Eng. 25 (1986) 1-116.

[16] Special Issue on Optical Processing for the Space Station, Opt. Eng. 25 (1986) 807-856.

[17] Special Issue on Photonic Computing, Appl. Opt. 25 (1986) 3019-3136.

[18] Special Issue on Optical Interconnexions, Opt. Eng. 25 (1986) 1075-1141.
[19] Special Issue on Optical Computing and Nonlinear Optical Signal Processing, Opt. Eng. 26 (1987) 159.

[20] Goodman, J. W., Dias, A. R. and Woody, L. M., Opt. Let. 2 (1978) 1-3.

[21] KUNG, H. T. and LeISERSON, C. E., in Introduction to VLSI, C.A. Mead and L.A. Conway, eds (Addison-Wesley, Reading) 1980.

[22] Lohmann, A. W., Stork, W. and Stucke, G., Appl. Opt. 25 (1986) 1530.

[23] Guilfoyle, P. S., Opt. Eng. 23 (1984) 20-25.

[24] Margolus, N., Physica 10D (1984) 81.

[25] Hopfield, J., Proc. Nat. Acad. Sc. 79 (1982) 2554.

[26] Psaltis, D. and Farhat, N., Opt. Let. 10 (1985) 98. 